\title{
Alk3/Alk3b and Smad5 Mediate BMP Signaling during Lymphatic Development in Zebrafish
}

\author{
Jun-Dae Kim ${ }^{1, *}$, and Jongmin $\mathrm{Kim}^{2, *}$
}

\begin{abstract}
Lymphatic vessels are essential to regulate interstitial fluid homeostasis and diverse immune responses. A number of crucial factors, such as VEGFC, SOX18, PROX1, FOX2C, and GJC2, have been implicated in differentiation and/or maintenance of lymphatic endothelial cells (LECs). In humans, dysregulation of these genes is known to cause lymphedema, a debilitating condition which adversely impacts the quality of life of affected individuals. However, there are no currently available pharmacological treatments for lymphedema, necessitating identification of additional factors modulating lymphatic development and function which can be targeted for therapy. In this report, we investigate the function of genes associated with Bone Morphogenetic Protein (BMP) signaling in lymphatic development using zebrafish embryos. The knock-down of BMP type II receptors, Bmpr2a and Bmpr2b, and type I receptors, Alk3 and Alk3b, as well as SMAD5, an essential cellular mediator of BMP signaling, led to distinct lymphatic defects in developing zebrafish. Therefore, it appears that each constituent of the BMP signaling pathway may have a unique function during lymphatic development. Taken together, our data demonstrate that BMP signaling is essential for normal lymphatic vessel development in zebrafish.
\end{abstract}

\section{INTRODUCTION}

Bone morphogenetic proteins (BMPs) are growth factors that belong to the tumor growth factor (TGF) superfamily and have been shown to regulate multiple biological processes of development and morphogenesis (David et al., 2009; Kawabata et al., 1998; Kondo, 2007; Sieber et al., 2009). Numerous BMP ligands, type I receptors (BMPRI/ALK), and type II receptors

\footnotetext{
${ }^{1}$ Yale Cardiovascular Research Center, Section of Cardiovascular Medicine, Department of Internal Medicine, Yale University School of Medicine, New Haven, CT 06511, USA, ${ }^{2}$ Department of Life Systems, Sookmyung Women's University, Seoul 140-742, Korea

*Correspondence: jun-dae.kim @yale.edu (JDK);

jkim@sookmyung.ac.kr (JK)
}

Received 9 January, 2014; accepted 28 January, 2014; published online 6 March, 2014

Keywords: bone morphogenetic protein (BMP) signal, lymphatic endothelial cells (LEC), lymphatic vessel development, SMAD, zebrafish
(BMPRII) have been identified in diverse experimental model systems and in the human genome (Beets et al., 2013; Guo and $\mathrm{Wu}, 2012)$. In general, BMP ligands signal throughout hetero-tetrameric receptor complexes, which consist of two BMPRIs and two BMPRIls. Upon activation, the BMP signaling complex undergoes Clathrin and Dab2 dependent internalization (Kim et al., 2012) and induces phosphorylation of receptor regulated SMADs (R-SMAD), such as SMAD1, 5, or 8 (9 in zebrafish), within the early endosomes (Hartung et al., 2006). Once phosphorylated, R-SMADs recruit a common mediator SMAD (CoSMAD), SMAD4, and translocate to the nucleus to promote transcription of BMP targets such as ID1 and SMAD6 (Guo and Wu, 2012).

During early embryogenesis, the activities of BMP signaling are important for dorso-ventral axis formation and the establishment of mesoderm-derived cell lineages (Kondo, 2007). Lack of functional BMP signaling in embryos leads to severe dorsalization, therefore, adversely affecting specification of ventral and mesodermal cell fates (Kondo, 2007; Little and Mullins, 2009; Sieber et al., 2009). During organogenesis, BMP signaling regulates morphogenesis of diverse mesoderm-derived organs. For instance, a reduced level of BMP signaling causes defects in the formation of heart primordial cells and cardiac valves (Abdelwahid et al., 2001; Chocron et al., 2007; Eisenberg and Markwald, 1995). In addition, increasing evidence suggests that BMP signaling also regulates morphogenesis of vascular networks by modulating behaviors of endothelial cells in vertebrates (Schmitt et al., 2013; Wiley and Jin, 2011; Wiley et al., 2011). In endothelial cells, BMP signaling can elicit opposite responses, depending on the type of ligand; while BMP2 and BMP6 promote angiogenesis (Finkenzeller et al., 2012; Wiley et al., 2011), BMP9 and BMP10 are known to induce quiescence of endothelial cells (Larrivée et al., 2012; Moya et al., 2012). More recently, BMP and TGF $\beta$ signaling have been shown to regulate lymphatic endothelial cells (LECs) (Dunworth et al., 2013; Kinashi et al., 2013; Levet et al., 2013; Yoshimatsu et al., 2013), which are specialized endothelial cells derived from endothelial cells within blood vessels. However, since BMP signaling can elicit drastically distinct outcomes in the same tissue dependent on the context (Kim et al., 2012; Wiley et al., 2011) and there is a high degree of redundancy within the signaling pathways (Guo and Wu, 2012; Little and Mullins, 2009), it is possible that BMP signaling differently regulates LECs in a context-dependent manner. For instance, BMP ligands may bind to distinct Type I receptors (i.e. Alk2 vs Alk3), or preferentially activate distinct R-SMADs (i.e. SMAD1 vs SMAD5) to 
Table 1. Morpholinos (MO) used in this paper

\begin{tabular}{lccc}
\hline Zebrafish gene & Sequence & MO type & References \\
\hline bmpr2a & 5'-CCTCTTACCTCAGTTACAATTTATA-3' & Negative control & This study (gene tools) \\
bmpr2b & 5'-TCATTACGGAAACATACCTCTTAGC-3' & Splicing blocking & Wiley et al. (2011) \\
ak1/acvr11 & 5'-AGTTGATTCTGACCTTGTTTACCA-3' & Splicing blocking & Wiley et al. (2011) \\
alk2/acvr1l & 5'-CTGCGAGCATCACTGAAGCCTTC-3' & Translation blocking & Roman et al. (2002) \\
alk3/bmpr1aa & 5'-GATTCATGTTTGTGTTCAATTTCCG-3' & Translation blocking & Bauer et al. (2001) \\
alk3b/bmpr1ab & 5'-GACGCATTGTCAAATTGTCTTGTCG-3' & Translation blocking & Little et al. (2009) \\
smad1 & 5'-GTCGAGTTGTTGAACTGTATGGCTG-3' & Translation blocking & Little et al. (2009) \\
smad5 & 5'-TAACAATTTAGCCACGCTCACCTGG-3' & Splicing blocking & McReynolds et al. (2007) \\
smad9 & 5'-ATCAGTGAAACCTACCTGGACTTTC-3' & Splicing blocking & This study \\
\hline
\end{tabular}

either activate or repress lymphatic development.

Therefore, we examined the function of individual BMP signaling components during lymphatic development using zebrafish as a model system. We found that many genes which function within the BMP signaling pathway, including bmpr2, bmpr1, bmpr1b, and smad5 are essential to promote lymphatic development in zebrafish. In contrast, the functions of alk2, smad1, and smad9 appear to be dispensable for early lymphatic development. Therefore, it appears that BMP signaling may promote lymphatic development via BMPR1/Alk3 and SMAD5 in zebrafish. Combined with our previous analyses on the role of BMP2 signaling in lymphatic development (Dunworth et al., 2013), our data presented here illustrate the complex and context-dependent nature of BMP signaling during lymphatic development.

\section{MATERIALS AND METHODS}

\section{Zebrafish husbandry and microinjection}

Zebrafish (Danio rerio) Tg(fli1a:nEGFP $)^{47} ; \operatorname{Tg}(k d r l: m C h e r r y)^{5843}$ transgenic embryos and adults were raised and maintained under IACUC guidelines of Yale university. Sequences of morpholino anti-sense oligonucleotides (MO; Gene Tools, LLC) can be found in Table 1. MOs were injected at 1-2 cell stage as desired concentrations. The efficacy of the MO was validated by semi-quantitative reverse transcriptase (RT) PCR.

\section{Morphological analysis and quantification of lymphatic phenotype}

Zebrafish embryos were anesthetized, plated and oriented laterally on a glass bottom dish at 4dpf. Image acquisition from zebrafish embryos was achieved using a Nikon confocal microscope and merged Z-stack images by MBF ImageJ program. The number of LECs in developing thoracic ducts of zebrafish embryos was individually counted from the trunk region spanning 7 somites, from somite boundary 8 or 9 to 15, on Z-stacked confocal images. Experiments were performed in triplicate. Quantification graphs were generated by PRISM program. Results were evaluated by two-tailed and/or unpaired Student's $t$ test and each error bar represents the standard error of the mean (SEM).

\section{RESULTS}

BMPRII/BMPR2 is the main type II receptor for BMP ligands, although BMP ligands can bind to ActRII and ActRIIB (Beets et al., 2013; Wakefield and Hill, 2013; Wiley and Jin, 2011). In the zebrafish genome, two orthologs of human BMPR2, bmpr2a and $b m p r 2 b$, exist and are highly expressed in developing venous endothelial cells (Wiley et al., 2011). To define the function of individual Bmpr2s during lymphatic development, we first attenuated the level of Bmpr2a and Bmpr2b activities by antisense morpholino oligonucleotide (MO)-mediated knock-down in Tg(fli1a:nEGFP); Tg(kdrl:mCherry) transgenic zebrafish embryos. This double transgenic line allows us to visualize individual lymphatic endothelial cells (LECs), therefore, allowing us to precisely quantify the number of developing LEC in the thoracic duct (TD) at 4dpf (Fig. 1) (Kim et al., 2013; Yaniv et al., 2006). General morphology, heart beat rate, and development of blood vessels in embryos injected with a $3.6 \mathrm{ng} / \mathrm{embryo}$ dose of $\mathrm{MO}$ were comparable to control MO-injected embryos (data not shown and Figs. 1A and 1B). However, at 4dpf, the number of LECs in Bmp2a or Bmp2b-deficient embryos (1.45 \pm 0.28 for bmp2a $\mathrm{MO}$ and $3.64 \pm 1.11$ for bmp2a $\mathrm{MO}$ ) was substantially reduced compared to control embryos $(9.17 \pm 0.67)$ (Figs. 1C$1 \mathrm{E})$, indicating that function of Bmpr2 is essential for lymphatic vessel development in zebrafish. Considering previous reports on anti-lymphangiogenic effects of BMP2 signaling in zebrafish (Dunworth et al., 2013), it is seemingly paradoxical that attenuation of Bmpr2 function adversely affects lymphatic development. However, it is important to take into consideration that Bmpr2 is not only required for BMP2 signaling, but is also essential for mediating pro-lymphangiogenic BMP9 signaling (Levet et al., 2013). In addition, it is possible that MO-mediated knock-down potentially affects venous endothelial cells, therefore, may indirectly influence LEC development. Regardless, our data illustrate Bmpr2 is essential for lymphatic development.

During BMP signal transduction, BMP type I receptors (BMPRIs, also known as Alks) are activated by BMP type II receptors upon ligand binding. While various TGF/BMP ligands may bind to BMP type II receptors, BMPRIs appear to retain certain ligand specificity or preference. Therefore, BMPRI most likely determines the down-stream effects and signaling outcomes of BMP signaling (David et al., 2009; Wiley and Jin, 2011). While ALK2/ACVR1, ALK3/BMPR1A and ALK6/BMPR1B mediate the signal transduction of BMP subfamily ligands and induce phosphorylation of SMAD1, 5, 9, ALK1/ACVRL1 appears to relay signaling of both TGF and BMP subfamily ligands (Ehrlich et al., 2011; Hartung et al., 2006; Miyazono et al., 2010; Wiley and Jin, 2011). To delineate the contribution of each BMP type I receptor in the formation of lymphatic vessel, we attenuated the level of Alk1, Alk2, Alk3, and Alk3b activities in zebrafish. Consistent with a previous report (Little and Mullins, 2009), the injections of 

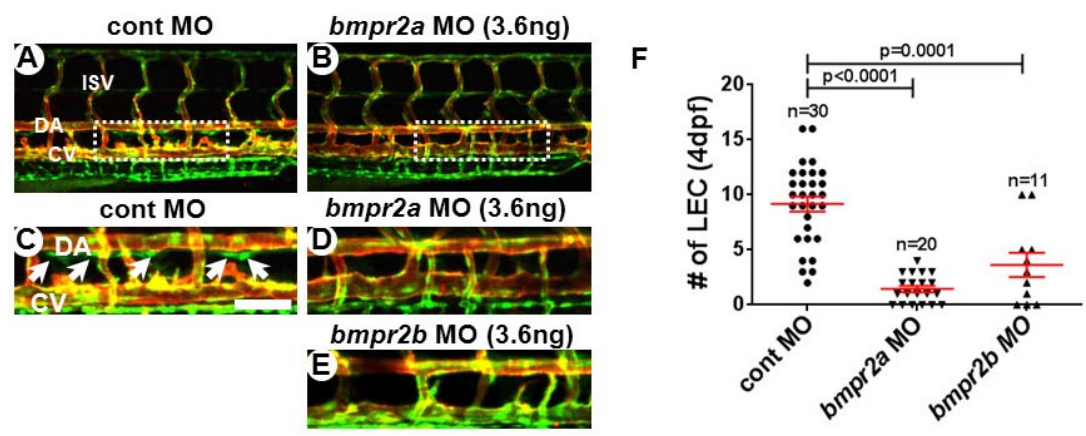

Fig. 1. Reduction in Bmpr2a and $2 b$ activity causes loss of lymphatic endothelial cells in thoracic duct of zebrafish. Confocal images taken from the trunk region of $4 \mathrm{dpf}$ control $(\mathrm{A}$, $\mathrm{C})$, bmpr2a (B, D), and bmpr2b (C) MOinjected embryos in Tg(fli1a:negfp); $T g(k d r l$ : $m$ Cherry) double transgenic background. $\mathrm{GFP}^{+}$mCherry cells are the lymphatic endothelial cells (LECs) within the thoracic duct (white arrows). (F) Quantification on the number of LECs in control, bmpr2a, and bmpr2b MO-injected embryos. LECs in the TD between $8^{\text {th }}$ and $15^{\text {th }}$ somite were quantitated by confocal imaging analyses. Areas within the white rectangles in $(A)$ and $(B)$ are shown in higher magnification in (C) and (D). DA, dorsal aorta; CV, cardinal vein; ISV, intersegmental vessel. Scale bar is $50 \mu \mathrm{m}$.


Fig. 2. A reduced level of individual Bmp type I receptors (BMPRI/Alks) does not cause lymphatic defects in zebrafish embryos. Confocal images of $4 \mathrm{dpf}$ control (A), alk1 (B), alk2 (C), alk3 (D), and alk3b (E) MO-injected embryos in Tg(fli1a:negfp); $T g$ (kdrl:mCherry) double transgenic background. White arrows point LECs. (F) Quantification on the number of LECs in MO-injected embryos. DA, dorsal aorta; CV, cardinal vein. Scale bar is $50 \mu \mathrm{m}$.

MOs against alk1 (7 ng/embryo), alk2 (5.4 ng/embryo), alk3 (14 ng/embryo), or alk3b (3.6 ng/embryo) at high concentrations cause gastrulation defects, and other morphological abnormalities such as cardiac edema (data not shown). Therefore, to bypass the earlier requirement of Alks, we titrated the concentration of MO to determine the dose which does not affect early development and heart formation. At lower concentration, MOs against each Alk receptor did not cause any discernible abnormalities in axis formation, cardiac morphogenesis, blood vessel development, or lymphatic vessel formation (3.6 ng/embryo for alk1, $2.7 \mathrm{ng} / \mathrm{embryo}$ for alk2 MOs, $7 \mathrm{ng} / \mathrm{embryo}$ for alk3, and 1.8 ng/embryo for alk3b) (data not shown and Fig. 2). Thus, a partial reduction of each Alk appears to have negligible effects on lymphatic vessel development (Fig. 2). Since it is possible that BMPRIs have redundant roles during lymphatic development in zebrafish, we analyzed the lymphatic phenotype of embryos which were injected with a combination of MOs targeting two Alks. In case any two Alk receptors may function redundantly, injecting suboptimal doses of $\mathrm{MO}$ targeting both Alks together may create a synthetic phenotype, which cannot be observed when a single $\mathrm{MO}$ was injected with a suboptimal dose. When we injected alk2 and alk3 together at sub-optimal doses (1.4 ng/embryo for alk2 MO and 3.6ng/embryo for alk3) (Figs. 3A, 3B, and $3 E$ ), or alk2 and alk3b together (1.4 ng/embryo for alk2 MO and $0.9 \mathrm{ng} / \mathrm{embryo}$ for alk3b) (Figs. 3A, 3C, and 3E), lymphatic vessels in the injected embryos were comparable to control embryos, suggesting that Alk2 does not have any redundant role with Alk3 or Alk3b in lymphatic development. In contrast, co-injection of alk3 and alk3b MOs with a sup-optimal dosage caused a substantial loss of LECs in the developing TD at 4dpf (Figs. 3A, 3D, and 3E), suggesting that Alk3 and Alk3b may redundantly regulate lymphatic development in zebrafish.

Upon activation, the BMP receptor complex induces phosphorylation of SMAD1, 5, 8 (9 in zebrafish), which is collectively known as R-SMAD (R-Smad in zebrafish). Phosphorylated RSMADs translocate into the nucleus with Co-SMAD, and functions as a transcription factor to specific target genes (Kawabata et al., 1998; Wiley and Jin, 2011). It is known that each SMAD has distinct roles dependent on the context of BMP signaling (Dick et al., 1999; McReynolds et al., 2007; Müller et al., 1999). For example, Smad1 and Smad5 appear to have an opposite effect in hematopoiesis of zebrafish (McReynolds et al., 2007). While the number of blood cells is increased in Smad1-deficient embryos, hematopoiesis is substantially decreased in Smad5-deficient embryos (McReynolds et al., 2007). Therefore, we speculate that each Smad might function differently in LECs of zebrafish. To examine this notion, we first designed splice-blocking MOs to inhibit the endogenous smad mRNA processing which allows us to bypass the early requirement of these genes (MO efficacy was validated by semiquantitative RT-PCR in Figs. 4A and 4B). Injection of splicing $\mathrm{MO}$ against each smad did not cause any morphological defects such as abnormalities in axis formation, cardiac function, or formation of blood vessels (data not shown). However, the number of LECs within the TD in smad5 MO-injected embryos was drastically decreased $(3.64 \pm 0.70)$ compared to control embryos (8.04 \pm 0.624$)$. In contrast, we did not find any obvious decrease in the number of LECs in smad1 or smad9 MOinjected embryos (Figs. 4C-4G). Therefore, Smad5 appears to be the most critical downstream mediator for BMP signaling in developing LECs in zebrafish. 

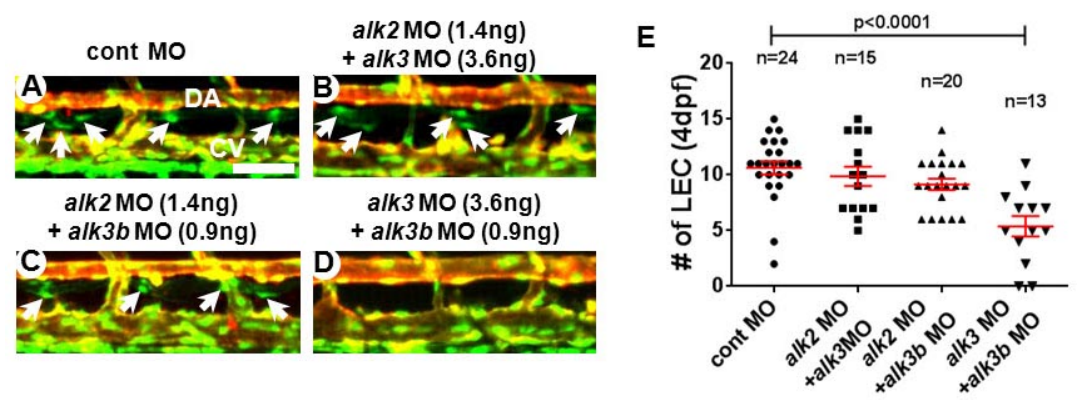

Fig. 3. Alk3 and Alk3b synergistically function to mediate Bmp signaling in lymphatic endothelial cells. Confocal images of $4 \mathrm{dpf}$ embryos injected with control $(A)$, alk2 and alk3 (B), alk2 and alk3b (C), and alk3 and alk3b (D) MOs. Attenuating alk3 and alk3b together drastically reduced the number of LECs in the TD. Arrows point LEC in TD. (E) Quantification on the number of LECs. DA, dorsal aorta; CV, cardinal vein. Scale bar is $50 \mu \mathrm{m}$.

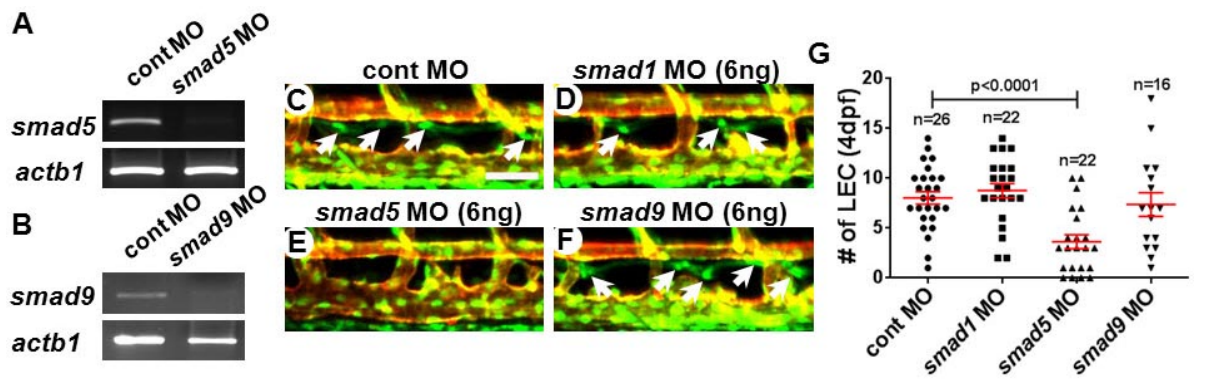

Fig. 4. Smad5 mediates the Bmp signaling in lymphatic endothelial cells in zebrafish. Validation of the MO efficacy by semi-quantitative RT-PCR. The transcripts of smad5 (A) and smad9 (B) were significantly decreased in embryos injected with $\mathrm{MO}$ against smad5 $(\mathrm{A})$ and smad9 (B). Confocal images of 4dpf embryos injected with control (C), smad1 (D), smad5 (E), or smad9 (F) MOs. Only smad5 MO-injected embryos show discernible changes in the number of LECs. Arrows point LECs in the TD. (G) Quantification on the number of LECs. DA, dorsal aorta; CV, cardinal vein; ISV, interseg-mental vessel. Scale bar is $50 \mu \mathrm{m}$.

\section{DISCUSSION}

Our data demonstrate that BMP signaling is essential for developing lymphangiogenesis in zebrafish. Among BMP Type I receptors, combined function of Alk3 and Alk3b appear to be essential for lymphatic development, while Alk2 appears to be largely dispensable for this process. In addition, Smad5, but not Smad1 or Smad9, is required to mediate BMP signaling within LECs. Our findings are consistent with recent findings which suggest the importance of BMP signaling in LECs (Dunworth et al., 2013; Farnsworth et al., 2011; Levet et al., 2013; Yoshimatsu et al., 2013). For instance, the binding of BMP9 to ALK1 receptors inhibits lymphangiogenesis and regulates lymphatic valve formation and maturation of LECs (Levet et al., 2013; Yoshimatsu et al., 2013). In addition, our recent data shows that the over-expression of Bmp2b at 48hpf in zebrafish decreases the number of LECs, illustrating the anti-lymphangiogenic activity of BMP2 signaling in lymphatic vessel formation (Dunworth et al., 2013). Therefore, as in the case of blood vessels (Kim et al., 2012; Larrivée et al., 2012; Moya et al., 2012; Wiley et al., 2011), it appears that BMP signaling may modulate development and/or maintenance of lymphatic vessels in a context-dependent manner. Considering the complex regulation of BMP signaling in other systems (Collery and Link, 2011; David et al., 2009; Farnsworth et al., 2011; Hartung et al., 2006; Miyazono et al., 2010), it is seemingly possible that distinct BMP ligands, of which distribution is spatiotemporally regulated, may exert pro- or anti-lymphangiogenic effects during development. Our analyses identify Smad5 as the most important downstream mediator of BMP signaling during lymphatic development. Considering that the majority of BMP signaling eventually converges at SMAD1, 5, 8/9, delineating how SMAD5 can distinguish activation by pro-lymphangiogenic BMP ligands (i.e. BMP9) and anti-lymphangiogenic BMP ligands (i.e. BMP2) may help us to better understand highly complex effects of BMP signaling in LECs.

\section{ACKNOWLEDGMENTS}

We thank Dr. Jose Cardona-Costa for excellent fish care and the Korea Zebrafish Organogenesis Mutant Bank (ZOMB) and ZFIN for providing zebrafish lines. This work has been supported by grants from American Heart Association post-doctoral fellowship to J.-D.K (11POST7440010) and this research was supported by Basic Science Research Program through the National Research Foundation of Korea (NRF) funded by the Ministry of Science, ICT \& Future Planning (NRF-2013R1A1A1 057591).

\section{REFERENCES}

Abdelwahid, E., Rice, D., Pelliniemi, L.J., and Jokinen, E. (2001). Overlapping and differential localization of Bmp-2, Bmp-4, Msx-2 and apoptosis in the endocardial cushion and adjacent tissues of the developing mouse heart. Cell Tissue Res. 305, 67-78.

Bauer, H., Lele, Z., Rauch, G.J., Geisler, R., and Hammerschmidt, M. (2001). The type I serine/threonine kinase receptor Alk8/Lost$\mathrm{a}-\mathrm{fin}$ is required for $\mathrm{Bmp} 2 \mathrm{~b} / 7$ signal transduction during dorsoventral patterning of the zebrafish embryo. Development 128 , 849-858.

Beets, K., Huylebroeck, D., Moya, I.M., Umans, L., and Zwijsen, A. (2013). Robustness in angiogenesis: notch and BMP shaping waves. Trends Genet. 29, 140-149.

Chocron, S., Verhoeven, M.C., Rentzsch, F., Hammerschmidt, M., and Bakkers, J. (2007). Zebrafish Bmp4 regulates left-right asymmetry at two distinct developmental time points. Dev. Biol. 305, 577-588.

Collery, R.F., and Link, B.A. (2011). Dynamic smad-mediated BMP signaling revealed through transgenic zebrafish. Dev. Dyn. 240, 712-722.

David, L., Feige, J.J., and Bailly, S. (2009). Emerging role of bone morphogenetic proteins in angiogenesis. Cytokine Growth Fac- 
tor Rev. 20, 203-212

Dick, A., Meier, A., and Hammerschmidt, M. (1999). Smad1 and Smad5 have distinct roles during dorsoventral patterning of the zebrafish embryo. Dev. Dyn. 216, 285-298.

Dunworth, W.P., Cardona-Costa, J., Cagavi, E., Kim, J.D., Fischer J.C., Meadows, S., Wang, Y., Cleaver, O., Qyang, Y., Ober, E. A., et al. (2013). Bone morphogenetic protein 2 signaling negatively modulates lymphatic development in vertebrate embryos. Circ. Res. 114, 56-66.

Ehrlich, M., Horbelt, D., Marom, B., Knaus, P., and Henis, Y.I. (2011). Homomeric and heteromeric complexes among TGF- $\beta$ and BMP receptors and their roles in signaling. Cell Signal. 23, 1424-1432.

Eisenberg, L.M., and Markwald, R.R. (1995). Molecular regulation of atrioventricular valvuloseptal morphogenesis. Circ. Res. 77, 1-6.

Farnsworth, R.H., Karnezis, T., Shayan, R., Matsumoto, M., Nowell, C.J., Achen, M.G., and Stacker, S.A. (2011). A role for bone morphogenetic protein-4 in lymph node vascular remodeling and primary tumor growth. Cancer Res. 71, 6547-6557.

Finkenzeller, G., Hager, S., and Stark, G.B. (2012). Effects of bone morphogenetic protein 2 on human umbilical vein endothelial cells. Microvasc. Res. 84, 81-85.

Guo, J., and Wu, G. (2012). The signaling and functions of heterodimeric bone morphogenetic proteins. Cytokine Growth Factor Rev. 23, 61-67.

Hartung, A., Bitton-Worms, K., Rechtman, M.M., Wenzel, V., Boergermann, J.H., Hassel, S., Henis, Y.I., and Knaus, P. (2006). Different routes of bone morphogenic protein (BMP) receptor endocytosis influence BMP signaling. Mol. Cell. Biol. 26, 77917805.

Kawabata, M., Imamura, T., and Miyazono, K. (1998). Signal transduction by bone morphogenetic proteins. Cytokine Growth Factor Rev. 9, 49-61.

Kim, J.D., Kang, H., Larrivée, B., Lee, M.Y., Mettlen, M., Schmid, S.L., Roman, B.L., Qyang, Y., Eichmann, A., and Jin, S.W. (2012). Context-dependent proangiogenic function of bone morphogenetic protein signaling is mediated by disabled homolog 2 . Dev. Cell 23, 441-448.

Kim, J.D., Kang, Y., Kim, J., Papangeli, I., Kang, H., Wu, J., Park H., Nadelmann, E., Rockson, S.G., Chun, H.J., et al. (2013). Essential role of apelin signaling during lymphatic development in Zebrafish. Arterioscler. Thromb. Vasc. Biol. 34, 338-345.

Kinashi, H., Ito, Y., Mizuno, M., Suzuki, Y., Terabayashi, T., Nagura, F., Hattori, R., Matsukawa, Y., Mizuno, T., Noda, Y., et al. (2013) TGF- $\beta 1$ promotes lymphangiogenesis during peritoneal fibrosis. J. Am. Soc. Nephrol. 24, 1627-1642.

Kondo, M. (2007). Bone morphogenetic proteins in the early development of zebrafish. FEBS J. 274, 2960-2967.

Larrivée, B., Prahst, C., Gordon, E., del Toro, R., Mathivet, T., Duarte, A., Simons, M., and Eichmann, A. (2012). ALK1 signaling inhibits angiogenesis by cooperating with the Notch pathway. Dev. Cell 22, 489-500.
Levet, S., Ciais, D., Merdzhanova, G., Mallet, C., Zimmers, T.A., Lee, S.J., Navarro, F.P., Texier, I., Feige, J.J., Bailly, S., et al. (2013). Bone morphogenetic protein 9 (BMP9) controls lymphatic vessel maturation and valve formation. Blood 122, 598-607.

Little, S.C., and Mullins, M.C. (2009). Bone morphogenetic protein heterodimers assemble heteromeric type I receptor complexes to pattern the dorsoventral axis. Nat. Cell Biol. 11, 637-643.

McReynolds, L.J., Gupta, S., Figueroa, M.E., Mullins, M.C., and Evans, T. (2007). Smad1 and Smad5 differentially regulate embryonic hematopoiesis. Blood 110, 3881-3890

Miyazono, K., Kamiya, Y., and Morikawa, M. (2010). Bone morphogenetic protein receptors and signal transduction. J. Biochem. 147, 35-51

Moya, I.M., Umans, L., Maas, E., Pereira, P.N., Beets, K., Francis, A., Sents, W., Robertson, E.J., Mummery, C.L., Huylebroeck, D. et al. (2012). Stalk cell phenotype depends on integration of Notch and Smad1/5 signaling cascades. Dev. Cell 22, 501-514.

Müller, F., Blader, P., Rastegar, S., Fischer, N., Knöchel, W., and Strähle, U. (1999). Characterization of zebrafish smad1, smad2 and smad5: the amino-terminus of smad1 and smad5 is required for specific function in the embryo. Mech. Dev. 88, 73-88.

Roman, B.L., Pham, V.N., Lawson, N.D. Kulik, M., Childs, S., Lekven, A.C., Garrity, D.M., Moon, R.T., Fishman, M.C., Lechleider, R.J., et al. (2002). Disruption of acvrl1 increases endothelial cell number in zebrafish cranial vessels. Development 129, 3009 3019.

Schmitt, C.E., Woolls, M.J., and Jin, S.W. (2013). Mutant-specific gene expression profiling identifies SRY-related HMG box 11b (SOX11b) as a novel regulator of vascular development in zebrafish. Mol. Cells 35, 166-172.

Sieber, C., Kopf, J., Hiepen, C., and Knaus, P. (2009). Recent advances in BMP receptor signaling. Cytokine Growth Factor Rev. 20, 343-355.

Wakefield, L.M., and Hill, C.S. (2013). Beyond TGF $\beta$ : roles of other TGF $\beta$ superfamily members in cancer. Nat. Rev. Cancer 13 , 328-341.

Wiley, D.M., and Jin, S.W. (2011). Bone Morphogenetic Protein functions as a context-dependent angiogenic cue in vertebrates. Semin. Cell Dev. Biol. 22, 1012-1018.

Wiley, D.M., Kim, J.D., Hao, J., Hong, C.C., Bautch, V.L., and Jin S.W. (2011). Distinct signalling pathways regulate sprouting angiogenesis from the dorsal aorta and the axial vein. Nat. Cell Biol. 13, 686-692.

Yaniv, K., Isogai, S., Castranova, D., Dye, L., Hitomi, J., and Weinstein, B.M. (2006). Live imaging of lymphatic development in the zebrafish. Nat. Med. 12, 711-716.

Yoshimatsu, Y., Lee, Y.G., Akatsu, Y., Taguchi, L., Suzuki, H.I., Cunha, S.I., Maruyama, K., Suzuki, Y., Yamazaki, T., Katsura, A., et al. (2013). Bone morphogenetic protein-9 inhibits lymphatic vessel formation via activin receptor-like kinase 1 during development and cancer progression. Proc. Natl. Acad. Sci. USA $110,18940-18945$ 